\title{
New Amber Mutation in a $\beta$-Thalassemic Gene with Nonmeasurable Levels of Mutant Messenger RNA In Vivo
}

\author{
George F. Atweh, Howard E. Brickner, Xiao-Xiang Zhu, Haig H. Kazazian, Jr., and Bernard G. Forget \\ Division of Hematology and Oncology, Department of Medicine, University of Michigan School of Medicine and Ann Arbor Veterans \\ Administration Hospital, Ann Arbor, Michigan 48105; Genetics Unit, Department of Pediatrics, The Johns Hopkins University School \\ of Medicine, Baltimore, Maryland 21205; and the Hematology Section, Department of Medicine, Yale University School of Medicine, \\ New Haven, Connecticut 06510
}

\begin{abstract}
We have identified a $\boldsymbol{\beta}$-thalassemia gene that carries a novel nonsense mutation in a Chinese patient. This mutation, a $\mathbf{G}$ to $T$ substitution at the first position of codon 43 , changes the glutamic acid coding triplet (GAG) to a terminator codon (TAG). Based on oligonucleotide hybridization studies of 78 Chinese and Southeast Asian $\beta$-thalassemia chromosomes, we estimate that this mutation accounts for a small minority of the $\beta$-thalassemia mutations in that population. Study of the expression of this cloned gene in a transient expression system demonstrated a $65 \%$ decrease in levels of normally spliced mutant $\beta$-globin mRNA. However, the study of reticulocyte RNA isolated from an individual heterozygous for this mutation demonstrated a total absence of this mutant mRNA in vivo. The basis for this big discrepancy between the level of accumulated mRNA in vivo and in vitro is probably the result of differences in the stabilities of the mutant mRNA in erythroid cells.
\end{abstract}

\section{Introduction}

The thalassemias are disorders of hemoglobin production characterized by imbalanced synthesis of $\alpha$ and $\beta$ chains. A large number of mutations that interfere with mRNA transcription, splicing, polyadenylation, or translation have been shown to result in a thalassemic phenotype (1). We have studied a $\beta$-thalassemia gene from a Chinese patient doubly heterozygous for $\beta$-thalassemia and a nondeletion $\beta$-thalassemia (2) by molecular cloning and expression in a heterologous transient expression system. An amber mutation at amino acid 43 was created by a $G$ to $T$ transversion. In addition to its deleterious effect on mRNA translation, this mutation seems to have a more profound effect on the level of mutant $\beta$-globin mRNA in vivo than in vitro. Study of reticulocyte RNA from an individual heterozygous for this mutation demonstrated a total absence of this mutant mRNA in vivo. These findings are discussed in relation to other $\beta$-thalassemia genes with different nonsense mutations.

This work was partially presented as an abstract in the 28th Annual Meeting of the American Society of Hematology in San Francisco, CA, 1986.

Address all correspondence to Dr. G. F. Atweh, Veterans Administration Medical Center, Hematology Section (111E), 2215 Fuller Road, Ann Arbor, MI 48105.

Received for publication 30 October 1987 and in revised form 2 February 1988.

The Journal of Clinical Investigation, Inc.

Volume 82, August 1988, 557-561

\section{Methods}

Structural studies of the mutant $\beta$-globin gene. High molecular weight DNA was isolated from the spleen of an individual who was double heterozygous for $\beta$-thalassemia and a nondeletion type of $\delta \beta$-thalassemia (2). The $\beta$-thalassemia allele could be distinguished from the nondeletion type of $\delta \beta$-thalassemia allele by the absence of the Ava II restriction site in $\beta$-IVS- 2 (2). We cloned the $\beta$-thalassemia gene as a $7.5 \mathrm{~kb}$ Hind III fragment in Charon 28 bacteriophage (3) and subcloned it as a $3.7 \mathrm{~kb}$ Pst $1 / \mathrm{Bgl}$ II fragment in the expression vector $\pi$ SVplac (4). DNA sequence analysis was performed by the dideoxy chain termination method of Sanger et al. (5) as described earlier (3). Oligonucleotides (19 mer), specific for the codon 43 mutation (CAGAGGTTCTTTTAGTCCT) and the normal sequence around codon 43 (GGTTCTTT GAGTCCTTTGG), were synthesized on a DNA synthesizer (Applied Biosystems, Inc., Foster City, CA). 10- $\mu \mathrm{g}$ samples of genomic DNA of various subjects were subjected to oligonucleotide hybridization analysis as previously described $(6,7)$.

Functional studies of the mutant $\beta$-globin gene. $\pi \mathrm{SV}$ plac recombinants containing the cloned mutant $\beta$-globin gene described above or a normal $\beta$-globin gene were cotransfected with an $\alpha$-globin gene into HeLa cells at $25 \%$ confluency using the technique of DNA coprecipitation with calcium phosphate followed by glycerol shock as described earlier (8). After $36 \mathrm{~h}$, the cells were harvested and cytoplasmic RNA was extracted using methods described by Favaloro et al. (9). The extracted RNA was used in S1 nuclease mapping experiments $(10,11)$ with either a $5^{\prime}$ end-labeled double-stranded probe or a uniformly labeled single-stranded probe (see Results for description of specific probes). After S1 nuclease digestion, the protected DNA fragments were fractionated by electrophoresis in 6 or $8 \%$ polyacrylamide gels in the presence of $7 \mathrm{M}$ urea. These same RNA, along with RNA isolated from reticulocytes of an individual heterozygous for this $\beta$-thalassemia, and another normal individual were used in RNase A protection assays. Uniformly labeled RNA probes were synthesized as described by Melton et al. (12) and hybridized to either $20 \mu \mathrm{g}$ of transfected HeLa cell RNA or $5 \mu \mathrm{g}$ of reticulocyte RNA at $52^{\circ} \mathrm{C}$ in a hybridization buffer made of $80 \%$ formamide, $0.4 \mathrm{M} \mathrm{NaCl}, 1 \mathrm{mM}$ EDTA, and $40 \mathrm{mM}$ Pipes, $\mathrm{pH}$ 6.7. The RNA/RNA hybrids were digested with $10 \mu \mathrm{g}$ RNase $A$ in $300 \mathrm{mM} \mathrm{NaCl}, 5 \mathrm{mM}$ EDTA, and $10 \mathrm{mM}$ Tris, $\mathrm{pH} 7.5$ at $30^{\circ} \mathrm{C}$ for $30 \mathrm{~min}$. The protected RNA fragments were also fractionated by electrophoresis in $6 \%$ polyacrylamide gels in the presence of $7 \mathrm{M}$ urea.

\section{Results}

The DNA sequence of the cloned $\beta$-thalassemia gene was determined from a position 100 nucleotides (nt) ${ }^{1}$ upstream from the cap site to $200 \mathrm{nt}$ downstream from the polyadenylation site excluding about $500 \mathrm{nt}$ in the middle of intron 2 . When the DNA sequence of the cloned $\beta$-thalassemia gene was compared with the sequence of a normal $\beta$-globin gene (13), five nucleotide substitutions were detected. Four of these have

1. Abbreviations used in this paper: AT, adenosine thymidine; nt, nucleotides. 
been previously demonstrated to represent polymorphic variations at codon 2 and IVS-2 positions 16, 74, and 666 (14). These polymorphisms define the $\beta$-globin gene framework as 3 Asian. In addition, a previously unidentified $\mathrm{G}$ to $\mathrm{T}$ transversion at the first position of codon 43 was detected (Fig. 1). This changes the glutamic acid coding triplet (GAG) to a stop codon (TAG) and destroys the ability of the mRNA to be translated into normal $\beta$-globin chains. This mutation falls within the recognition sequence of the restriction enzyme Hinf I and would be expected to lead to the loss of that restriction site. This was tested in a Southern blotting experiment where $10 \mu \mathrm{g}$ of DNA from the cloned gene was digested with the enzyme Hinf I, run on a $2 \%$ agarose gel and probed with a $3^{\prime}$ end-labeled DNA probe which extends from position 36 to 224 of exon 2 . When DNA from a normal cloned $\beta$-globin gene is used, a 188-nt fragment is detected (Fig. 2); when DNA from the cloned $\beta$-thalassemia gene is used, the $188-\mathrm{nt}$ fragment is lost and a larger 263-nt fragment is detected instead. This fragment reflects the loss of the Hinf I site predicted by the DNA sequence data.

A synthetic oligonucleotide that can detect this mutation was synthesized and used as a probe in hybridization experiments with genomic DNA, which was obtained from a patient who carries the only remaining uncharacterized $\beta$-thalassemia chromosome from a panel of $78 \beta$-thalassemia chromosomes from the Chinese and Southeast Asian population (15). We demonstrated that this patient, who was shown earlier by Chang et al. (16) to have a $\beta^{17}$ nonsense mutation on one of his $\beta$-globin alleles (15), has the same $\beta^{43}$ nonsense mutation described here on his other $\beta$-globin allele (data not shown). From these data, we estimate that this $\beta$-thalassemia mutation makes up a small minority of the $\beta$-thalassemia genes in Chinese and Southeast Asians.

To investigate the functional consequences of this mutation, we used a tranșient expression system where $\beta$-globin mRNA, which was transcribed from the mutant $\beta$-thalassemia gene that was transfected into HeLa cells, is compared with $\beta$-globin mRNA transcribed from a normal $\beta$-globin transfected into similar cells. A $5^{\prime}$ end labeled DNA probe extending from the Bam $\mathrm{HI}$ site in exon 2 to the Bam $\mathrm{H} 1$ site $5^{\prime}$ to the $\beta$-globin gene was used in an $\mathrm{S} 1$ nuclease mapping experiment. The RNA isolated from HeLa cells that were transfected with a normal $\beta$-globin gene protected the expected 209-nt fragment seen in Fig. 3, lane 2. When RNA, which was isolated from

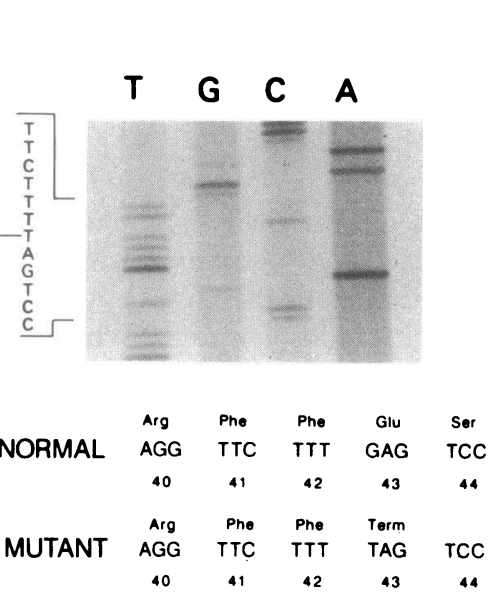

Figure 1. DNA sequence. Autoradiograph of a DNA sequencing gel representing sequences between codon 40 and codon 44 of the cloned $\beta$-thalassemia gene. The drumstick identifies the $\mathbf{G}$ to $T$ substitution at the first position of codon 43. The normal and mutant DNA sequences are denoted under the autoradiograph along with the corresponding amino acid sequence.

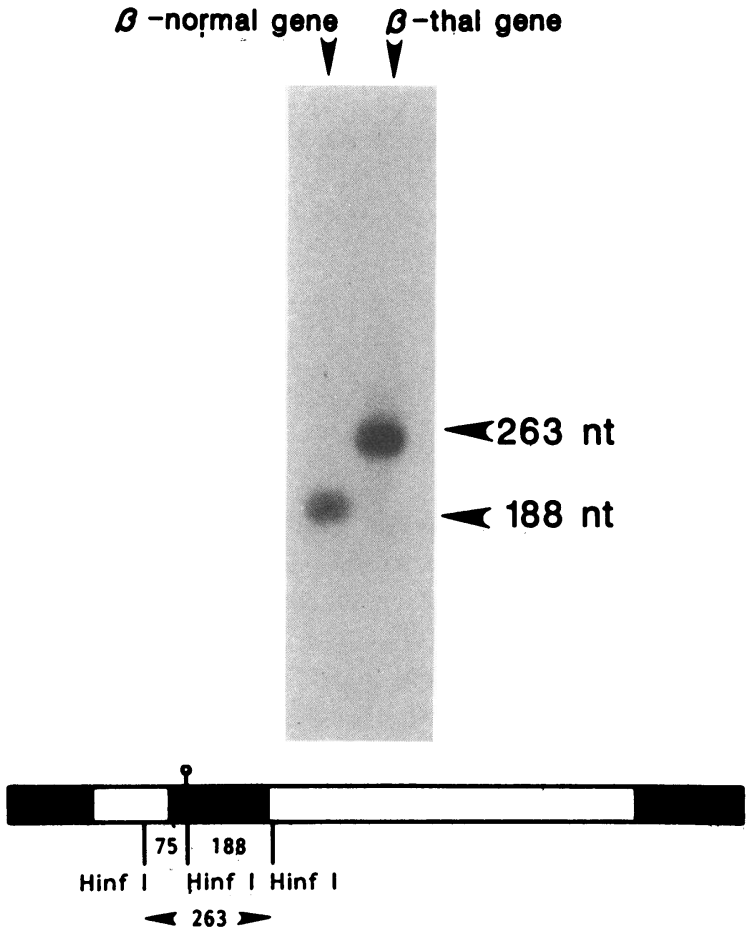

Figure 2. Demonstration of the mutation by Southern blotting. Autoradiograph of a Southern blot where a cloned normal $\beta$-globin gene and the cloned $\beta$-thalassemia gene were digested with Hinf I and probed with an exon 2 probe. A schematic map of a normal $\beta$-globin gene with the relevant Hinf I sites is shown under the autoradiograph. The mutation leads to the loss of the Hinf I site, which is marked by the drumstick, and the replacement of the 188-nt fragment seen in the normal gene with a 263-nt fragment in the thalassemic gene.

HeLa cells transfected with the $\beta$-thalassemia gene, was used in a similar experiment, the same 209-nt fragment was seen in addition to a new 171-nt fragment (Fig. 3, lane 1). This 171-nt fragment arises from the protection of a part of the probe which extends from the 5'-labeled end to the mutant nucleotide within exon 2 . This could be the result of either aberrant splicing at the site of the mutation or to recognition of the single base mismatch between the RNA and DNA by the S1 nuclease enzyme. To distinguish between these two possibilities, we repeated the $\mathrm{S} 1$ mapping study using a similar DNA probe made from mutant $\beta$-globin gene DNA instead of normal DNA. This probe is completely complementary to the mutant mRNA and has a single base mismatch with the normal mRNA. This probe was hybridized to the same RNA used in lanes 1 and 2 of Fig. 3. In this case, the mutant RNA protected a single 209-nt fragment (Fig. 3, lane 3) and the normal RNA protected a 209- and 171-nt fragment (Fig. 3, lane 4). This confirms the hypothesis that the 171-nt fragment is a result of the digestion of the probe DNA by $\mathrm{S} 1$ nuclease at the site of the mismatch. When a uniformly labeled probe generated from the mutant clone that spans the four intronexon junctions was made (3), splicing was shown to be normal at all intron-exon junctions (data not shown). When the level of $\beta$-globin mRNA in the HeLa cells transfected with the normal gene was compared to that of $\beta$-globin mRNA in HeLa cells transfected with the mutant $\beta$-globin gene, after correcting for differences in the efficiency of transfection by normal- 


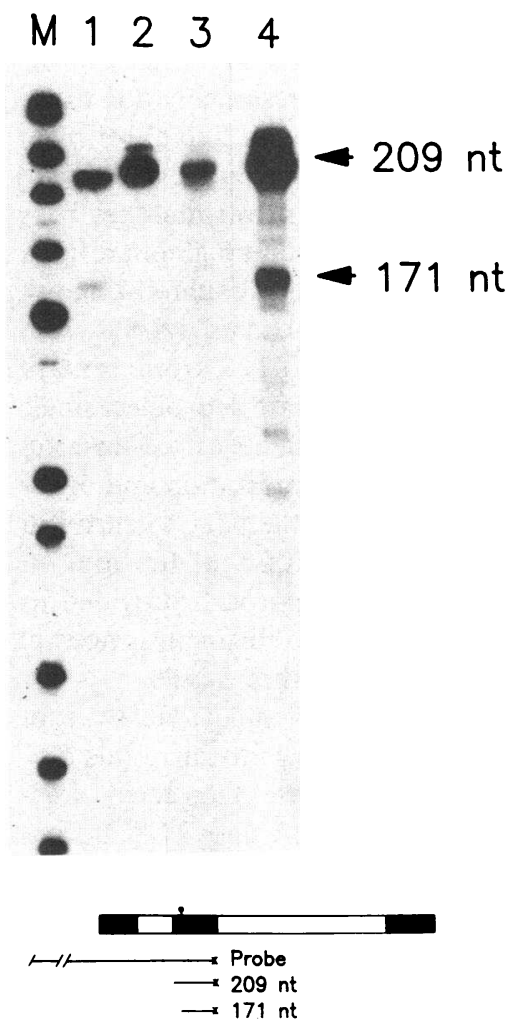

Figure 3. S1 mapping of mutant mRNA. S1 nuclease mapping study of RNA from HeLa cells transfected with either the $\beta$-thalassemia gene or a normal $\beta$-globin gene using a $5^{\prime}$ endlabeled probe, which is represented on the schematic map below the autoradiograph. The size marker $(M)$ consists of $3^{\prime}$ end-labeled Hpa II fragments of pBR322 plasmid DNA. In lanes 1 and 2 , the probe DNA was derived from a normal $\beta$-globin gene, and in lanes 3 and 4 , the probe DNA was derived from a $\beta^{43} \mathrm{mu}$ tant globin gene. Lane 1 shows the protected fragments when $\beta$-thalassemia mRNA is hybridized to normal probe DNA (mismatch at $\beta^{43}$ ), while in lane 2 , normal $\beta$-globin mRNA is hybridized to normal probe DNA (no mismatch). In lane 3, $\beta$-thalassemia mRNA is hybridized to $\beta$-thalassemia probe DNA (no mismatch), while in lane 4, normal $\beta$-globin mRNA is hybridized to $\beta$ thalassemia probe DNA (mismatch at $\beta^{43}$ ). While the expected 219 $\mathrm{nt}$ fragment is seen in every lane, a 171 -nt fragment is only seen in lanes where an mRNA/probe DNA mismatch occurs. This 171-nt fragment is most likely a result of $\mathrm{S} 1$ digestion of the probe at the site of the mutation.

izing to the level of expression of the cotransfected $\alpha$-globin gene, the mutant RNA level was determined to be $35 \%$ of the normal RNA.

In the absence of an individual homozygous for this mutation, it would be extremely difficult to determine the functional consequences of this mutation in erythroid cells in vivo. To circumvent this problem, we used a novel approach to differentiate the $\beta$-globin mRNA transcribed from the normal allele from the $\beta$-globin mRNA transcribed from the mutant allele in the reticulocyte RNA of the simple heterozygote available for this study. This approach was suggested to us by the ability of S1 nuclease to partially recognize the single base mismatch at the site of the mutation as shown in the experiment described above. We predicted that such a mismatch may be completely recognized by a different nuclease (RNase A) if an RNA probe is used instead of a DNA probe (12). We synthesized an antisense RNA extending from position -100 upstream from the cap site to position 49 of exon 3 and used it in the RNase protection experiment shown in Fig. 4. We were able to optimize the hybridization and digestion conditions to allow for $100 \%$ recognition of the mismatch between the mutant RNA and the normal sequence of the probe. Whereas the normal RNA protects the expected 223-, 143-, and 49-nt fragments (Fig. 4), the mutant RNA shows protection of the same 143- and 49-nt fragments and the complete replacement of the 223-nt fragment by a 185-nt fragment, which results from

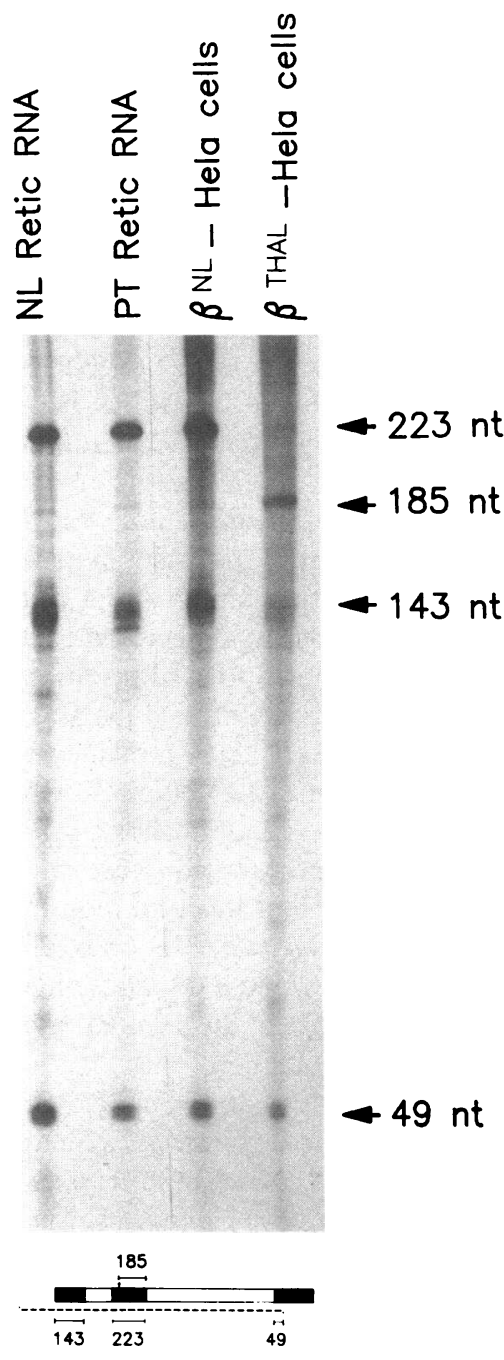

Figure 4. RNase A mapping of mutant mRNA. RNase A protection assay using an antisense radiolabeled RNA probe, which is represented on the schematic map of the $\beta$-globin gene below the autoradiograph. Lane 1 shows the protection by normal reticulocyte RNA of the 223-, 143-, and 49-nt fragments corresponding to exon 2 , exon 1, and part of exon 3 , respectively. Lane 2 shows the identical protected fragments that result from the use of reticulocyte RNA from the patient. Lane 3 also shows a similar protection pattern when RNA from HeLa cells that have been transfected with a normal $\beta$-globin gene is used. In lane 4, RNA from HeLa cells transfected with the $\beta$-thalassemia gene is used. The 223-nt fragment corresponding to exon 2 is completely replaced by a 185-nt fragment while the 143- and 49-nt fragments corresponding to exons 1 and 3 are unchanged. An expected 38-nt fragment, which results from cleavage of the 223-nt fragment to generate the 185-fragment, is too small to be seen on this autoradiograph. Very small amounts of the 185-nt fragment are also seen in every lane of the autoradiograph. This is probably due to overdigestion of the probe by RNase A in the AT-rich region around codon 43 .

complete digestion at the site of the mismatch (Fig. 4). Therefore, this provides us with an accurate and efficient way of distinguishing the mutant $\beta$-globin mRNA (185-nt fragment) from the normal $\beta$-globin mRNA (223-nt fragment) when the same conditions of hybridization and digestion are used.

We then attempted to quantitate the level of mutant $\beta$-globin mRNA in the reticulocyte RNA of the individual heterozygous for this mutation. When an RNase A protection experiment was performed comparing reticulocyte RNA from a normal individual with reticulocyte RNA from the affected individual under conditions that allow for $100 \%$ detection of the mismatch, no differences were noted between the two RNAs (Fig. 4). In both the normal and patient reticulocyte lanes, a faint 185 -nt band is seen that probably resulted from RNase A overdigestion at the adenosine thymidine (AT)-rich region around codon 43. However, since the normal reticulocyte RNA and the patient's RNA show an identical protection pattern, we can conclude that the level of mutant mRNA in vivo must be extremely low. 


\section{Discussion}

In the course of the study of the molecular pathology of the $\beta$-globin cluster of a family from China, we detected a $\beta$-thalassemia globin gene on a chromosome with Chinese haplotype 2 $(2,15)$. Study of the hemoglobin phenotype in different family members did not allow for the unambiguous characterization of this mutant gene as a $\beta^{\circ}$-thalassemia or $\beta^{+}$-thalassemia gene. Previous studies of the spectrum of the $\beta$-thalassemia mutations in China and Southeast Asia showed that $\beta$-thalassemia genes on chromosomes of haplotype 2 may be associated with a frameshift 41-42 mutation, nonsense 17 mutation, $-28 \mathrm{~A}$ to $\mathrm{G}$ promoter mutation, or frameshift $71-72$ mutation (15). Oligonucleotide hybridization demonstrated the absence of any of these mutations from the $\beta$-globin gene on the haplotype 2 chromosome in this family (data not shown). Gene cloning and DNA sequence analysis revealed a novel nonsense mutation resulting from a $G$ to $T$ substitution at the first position of codon 43. In our panel of $78 \beta$-thalassemia genes from China and Southeast Asia, there remained a single uncharacterized $\beta$-globin gene on a haplotype 3 or 5 chromosome (15). Oligonucleotide hybridization using a synthetic oligonucleotide probe that can detect the nonsense mutation at codon 43 revealed the presence of this mutation in that $\beta$-globin gene. This completes the characterization of all the mutations in the available panel of Chinese $\beta$-thalassemia chromosomes and suggests a low incidence of the $\beta^{43}$ nonsense mutation in mutant genes from that population.

To study the functional effects of this mutation on $\beta$-globin mRNA metabolism, we used an in vitro expression system where the mutant $\beta$-thalassemia gene is cloned in an expression vector and introduced into heterologous HeLa cells. The mRNA expression of the mutant gene is then compared with that of a normal gene transfected in parallel into similar cells. Globin mRNA was accumulated in the steady state in those cells, and then quantitated and mapped by $\mathrm{S} 1$ nuclease analysis. HeLa cells that were transfected with the mutant $\beta^{43}$ gene accumulated $35 \%$ of the globin mRNA level in HeLa cells transfected with a normal $\beta$-globin gene. This is quite similar to the data of Takeshita et al. (17) and Humphries et al. (18) who studied a $\beta$-globin gene with a nonsense mutation at position 39 in a similar transient expression system. Baserga et al. studied the expression of several in vitro-generated $\beta$-globin mutant genes with nonsense mutations at codons $17,37,39$, and 82 , and found decreased accumulation of $\beta$-globin mRNA in cells transfected with all of those genes (19). Moschonas et al., however, detected the same level of $\beta$-globin mRNA in HeLa cells transfected with a normal gene or with a gene with the $\beta^{39}$ nonsense mutation (20). The reason for the discrepancy between the results of this study and all the other studies mentioned above is not clear.

A more relevant question is what happens in vivo in erythroid cells of individuals with the mutant gene described above. Such an analysis could have been made easier if an individual homozygous for this mutant allele were available for this study. In the absence of such an individual, one can only obtain information about the functional consequences of this mutation in vivo if the mRNA transcribed from the mutant allele could be distinguished from that transcribed from the other normal allele (in a simple heterozygote). Thus, it became imperative to develop an assay that can distinguish two mRNA species that differ at a single nucleotide. S1 nu- clease, which is notoriously inefficient at recognizing single base mismatches (21), was nonetheless able to partially recognize this mismatch as shown in Fig. 3, presumably due to the AT-rich sequence surrounding the mismatch. This experiment raised the possibility that using a different nuclease that was more efficient at recognizing single base mismatches may allow for the detection of $100 \%$ of the mutant $\beta$-globin mRNA and provide us with a quantitative assay that could distinguish normal and mutant mRNA. Winter et al. had used RNase $A$ to successfully distinguish normal K-ras RNA from mutant K-ras RNA (22) and Myers et al. used RNase A to detect single base substitutions in genomic DNA of patients with $\beta$-thalassemia (23). We were able to adjust the hybridization and digestion conditions of our RNase A protection assay to cleave at the site of this mismatch in close to $100 \%$ of the mutant mRNA molecules (Fig. 4). Thus, this approach provided us with a convenient assay that allows us to distinguish normal and mutant globin mRNA in vivo in erythroid cells.

When this assay was used to study reticulocyte RNA from the only surviving individual who is known to carry this mutant gene (simple heterozygote), the RNase A-protected fragments were identical in the patient's RNA and RNA from a normal control (Fig. 4). We estimate that the sensitivity of this assay should allow the detection of $3 \%$ or more of mutant RNA relative to normal RNA. Therefore, we may conclude that steady state mutant mRNA levels in erythroid cells in vivo are $<3 \%$ that of normal mRNA from the normal allele. It is interesting to note that the other patient, who was shown by oligonucleotide hybridization to carry the same mutant gene, is a double heterozygote for the $\beta^{17}$ and $\beta^{43}$ nonsense mutation. When reticulocyte RNA from this patient was used as a substrate in a cell-free translation assay in the presence of a serine-inserting amber suppressor tRNA, a $\beta^{171 y-s e r}$ polypeptide product was detected that corresponded to the $\beta^{17}$ mutant mRNA (24). No $\beta^{43}$ mutant mRNA was detected in the translation products of the patient's RNA (24). This may be the result of the presence of very low levels of $\beta^{43}$ mRNA in the reticulocyte RNA of the affected individual or conceivably, a result of the nonsuppressability of the amber mutation of the $\beta^{43}$ allele. Based on the calculations of Chang et al., all the globin mRNA in this patient could be accounted for by the $\beta^{17}$ allele. This led them to propose at that time that the other allele may contain a different mutation that results in the absence of mature $\beta$-globin mRNA from that gene (24). Their results, which were derived using a completely different approach in a different patient with the same mutation, are in agreement with ours.

It appears that the finding of low levels of $\beta$-globin mRNA in vivo, transcribed from genes with nonsense mutation, is a widespread phenomenon. It also appears that there is considerable variation in the levels of mutant mRNA in reticulocytes from patients with different nonsense mutations ranging from $<1 \%$ in a patient with a single nucleotide deletion at codon 44 , which produces a terminator at codon $60(25)$, to $15 \%$ in the patient with the $\beta^{17}$ nonsense mutation (24). There is no apparent linear relationship, however, between the level of mutant mRNA and the position of the mutation. The reasons for the decreased levels of mutant mRNA in vivo are still not clear, although differences in mRNA stability in erythroid cells is still the most attractive hypothesis. Different stabilities of globin mRNA in bone marrow cells and in HeLa cells were demonstrated by Maquat et al. in the $\beta$-thalassemia that re- 
sulted from a single nucleotide deletion at codon $44(26)$. The available experimental data suggest that missense mutations do not lead to similar decreases in levels of mutant mRNA (19) and that a relationship may exist between the ability to translate mRNA and its stability in vivo. The exact nature of the mechanism responsible for the low levels of mRNA in vivo remains to be elucidated.

\section{Acknowledgments}

The expert technical assistance of Laura Harmon and the secretarial assistance of Wilma Savitski are appreciated.

This work was supported in part by grants from the Veterans Administration and from the National Institutes of Health.

\section{References}

1. Bunn, H. F., and B. G. Forget. 1986. Hemoglobin: Molecular, Genetic and Clinical Aspects. Saunders, Philadelphia. 223-303.

2. Atweh, G. F., D. E. Zhu, and B. G. Forget. 1986. A novel basis for $\delta \beta$-thalassemia in a Chinese family. Blood. 68:1108-1113.

3. Atweh, G. F., N. P. Anagnou, J. Shearin, B. G. Forget, and R. E. Kaufman. 1985. $\beta$-thalassemia resulting from a single nucleotide substitution in an acceptor splice site. Nucleic Acids Res. 13:777-790.

4. Treisman, R., S. H. Orkin, and T. Maniatis. 1983. Specific transcription and RNA splicing defects in five cloned $\beta$-thalassemia genes. Nature (Lond.). 302:591-596.

5. Sanger, F., and A. R. Coulson. 1975. A rapid method for determining sequences in DNA by primed synthesis with DNA polymerase. J. Mol. Biol. 94:441-448.

6. Huang, S., C. Wong, S. E. Antonarakis, T. Ro-lien, W. H. Y. Lo, and H. H. Kazazian, Jr. 1986. The same "TATA" box $\beta$-thalassemia mutation in Chinese and US blacks: another example of independent origins of mutations. Hum. Genet. 74:162-164.

7. Studencki, A. B., and R. B. Wallace. 1984. Allele-specific hybridization using oligonucleotide probes of very high specific activity. Discrimination of the human $\beta^{\mathrm{A}}$ - and $\beta^{\mathrm{S}}$-globin genes. $D N A(N Y)$. 3:7-15.

8. Atweh, G. F., C. Wong, R. Reed, S. E. Antonarakis, D. E. Zhu, P. K. Ghosh, T. Maniatis, B. G. Forget, and H. H. Kazazian. 1987. A new mutation in IVS- 1 of the human $\beta$-globin gene causing $\beta$-thalassemia due to abnormal splicing. Blood. 70:147-151.

9. Favaloro, J., R. Treisman, and R. Kamen. 1980. Transcription maps of polyoma virus-specific RNA: analysis by two-dimensional nuclease S1 gel mapping. Methods Enzymol. 65:718-749.

10. Berk, A. J., and P. A. Sharp. 1977. Sizing and mapping of early adenovirus mRNAs by gel electrophoresis of $\mathrm{S} 1$ endonuclease digested hybrids. Cell. 12:721-732.

11. Weaver, R. F., and C. Weissman. 1979. Mapping of RNA by a modification of the Berk-Sharp procedure: the 5 ' termini of $15 S \beta$-globin mRNA precursor and mature 10S $\beta$-globin mRNA have identical map coordinates. Nucleic Acids Res. 7:1175-1193.
12. Melton, D. A., P. A. Kreig, M. R. Rebagliati, T. Maniatis, K. Zinn, and M. R. Green. 1984. Efficient in vitro synthesis of biologically active RNA and RNA hybridization probes from plasmids containing a bacteriophage SP6 promoter. Nucleic Acids Res. 12:70357055.

13. Lawn, R. M., A. Efstratiadis, C. O'Connell, and T. Maniatis. 1980. The nucleotide sequences of the human $\beta$-globin gene. Cell. 21:647-651.

14. Orkin, S. H., H. H. Kazazian, S. E. Antonarakis, S. C. Goff, C. D. Boehm, J. P. Sexton, P. G. Waber, and P. J. V. Giardina. 1982. $\beta$-thalassemia mutations, $\beta$-globin gene polymorphisms, and their linkage with restriction site polymorphisms in the human $\beta$-globin gene cluster. Nature (Lond.). 296:627-631.

15. Kazazian, H. H., Jr., C. E. Dowling, P. C. Waber, S. Huang, and W. H. Y. Lo. 1986. The spectrum of $\beta$-thalassemia genes in China and Southeast Asia. Blood. 68:964-967.

16. Chang, J. C., and Y. W. Kan. 1979. $\beta^{\circ}$-thalassemia, a nonsense mutation in man. Proc. Natl. Acad. Sci. USA. 76:2886-2889.

17. Takeshita, K., B. G. Forget, A. Scarpa, and E. J. Benz, Jr. 1984. Intranuclear defect in $\beta$-globin mRNA accumulation due to a premature translation termination codon. Blood. 64:13-22.

18. Humphries, R. K., T. J. Ley, N. P. Anagnou, A. W. Baur, and A. W. Nienhuis. 1984. $\beta^{\circ}-39$ thalassemia gene: a premature termination codon causes $\beta$-mRNA deficiency without affecting cytoplasmic $\beta$-mRNA stability. Blood. 64:23-32.

19. Baserga, S. J., A. Scarpa, and E. J. Benz, Jr. 1986. Five nonsense mutations in the human $\beta$-globin gene that also affect gene expression. Clin. Res. 34:447a.(Abstr.)

20. Moschonas, N., E. deBoer, F. G. Grosveld, H. H. Dahl, S. Wright, C. K. Shewmaker, and R. A. Flavell. 1981. Structure and expression of a cloned $\beta^{\circ}$-thalassemic globin gene. Nucleic Acids Res. 9:4391-4401.

21. Shenk, T. E., C. Rhodes, P. W. Rigby, and P. Berg. 1975. Biochemical method for mapping mutational alterations in DNA with S1 nuclease: the location of deletions and temperature-sensitive mutations in simian virus 40. Proc. Natl. Acad. Sci. USA. 72:989-993.

22. Winter, E., F. Yamamato, C. Almoguera, and M. Perucho. 1985. A method to detect and characterize point mutations in transcribed genes: amplification and overexpression of the mutant c-Ki-ras allele in human tumor cells. Proc. Natl. Acad. Sci. USA. 82:75757579.

23. Myers, R. M., Z. Larin, and T. Maniatis. 1985. Detection of single base substitutions by ribonuclease cleavage at mismatches in RNA:DNA duplexes. Science (Wash. DC). 230:1242-1246.

24. Chang, J. C., G. F. Temple, R. F. Trecartin, and Y. W. Kan. 1979. Suppression of the nonsense mutation in homozygous $\beta^{\circ}$ thalassemia. Nature (Lond.). 281:602-603.

25. Kinniburgh, A. J., L. E. Maquat, T. Schedl, E. Rachmilewitz, and J. Ross. 1982. mRNA-deficient $\beta^{\circ}$-thalassemia results from a single nucleotide deletion. Nucleic Acids Res. 10:5421-5427.

26. Maquat, L. E., and A. J. Kinniburgh. 1985. A $\beta^{\circ}$-thalassemic $\beta$-globin RNA that is labile in bone marrow cells is relatively stable in HeLa cells. Nucleic Acids Res. 13:2855-2867. 